\title{
Microfilaria Causing Bone Marrow Failure
}

\section{Sasidharan PK , Geetha P, Shaan M, Sreejith R and Aslam M}

Department of Medicine, Government Medical College, Kozhikode, Kerala, India

*Corresponding author: Sasidharan PK, Department of Medicine, Government Medical College, Kozhikode, Kerala, India, E-mail: sasidharanpk@gmail.com

Citation: Sasidharan PK, Geetha P, Shaan M, Sreejith R, Aslam M (2014) Microfilaria Causing Bone marrow failure. J Case Rep Stud 2(4): 403. doi: 10.15744/2348-9820.2.103

Received Date: April 24, 2014 Accepted Date: May 08, 2014 Published Date: August 13, 2014

\begin{abstract}
It is common to detect microfilariae in various cytological preparations, however there are very few case reports describing microfilariae in bone marrow aspirates. It is rarer to get bone marrow failure secondary to microfilaria in bone marrow. We report here a patient from Bihar, presented to us with prolonged fever and pancytopenia, bone marrow aspirate showed microfilaria. Treatment with DEC and albendazole resulted in prompt recovery.
\end{abstract}

Keywords: Microfilariae; Bone marrow failure; Pancytopenia

\section{Case Report}

Our patient is a 26 year old male, from Patna, Bihar now working in footware factory, in Kerala, presented with fever, headache and vomiting for 6 days. Fever was high grade, remittent with chills and rigor and headache was bifrontal, moderate in severity. He had occasional vomiting which was not projectile. There was no history of dysuria, respiratory or abdominal symptoms. There was no history of any bleeding manifestations. There was no significant illness in the past. He was addicted to tobacco chewing, was unmarried and there was no history of any high risk behavior. On examination, he was conscious, oriented, moderately built and nourished. He was febrile with stable vital signs. There was pallor, no icterus, clubbing or lymphadenopathy. Abdomen examination showed mild hepatosplenomegaly which was firm and non tender, without any free fluid. All other systems were within normal limits.

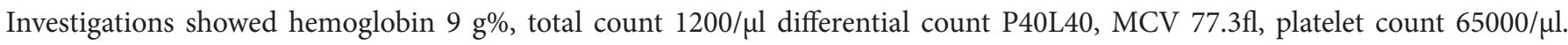
His liver, renal function tests, serum electrolytes, blood sugar were within normal limits. Initially a possibility of malaria was considered and patient was started on chloroquine and primaquine without any response as fever persisted. Peripheral smear for malaria parasites was repeatedly negative. Artesunate and Doxycycline was added considering falciparum malaria, but fever was persisting. Meanwhile his total count dropped to $700 / \mu \mathrm{l}$ and hemoglobin dropped to $6.5 \mathrm{~g} \%$. Suspecting underlying bone marrow failure, bone marrow aspirate was done which showed microfilaria (Figure -1,2) and the cellularity was decreased, reported as ? hypoplastic.

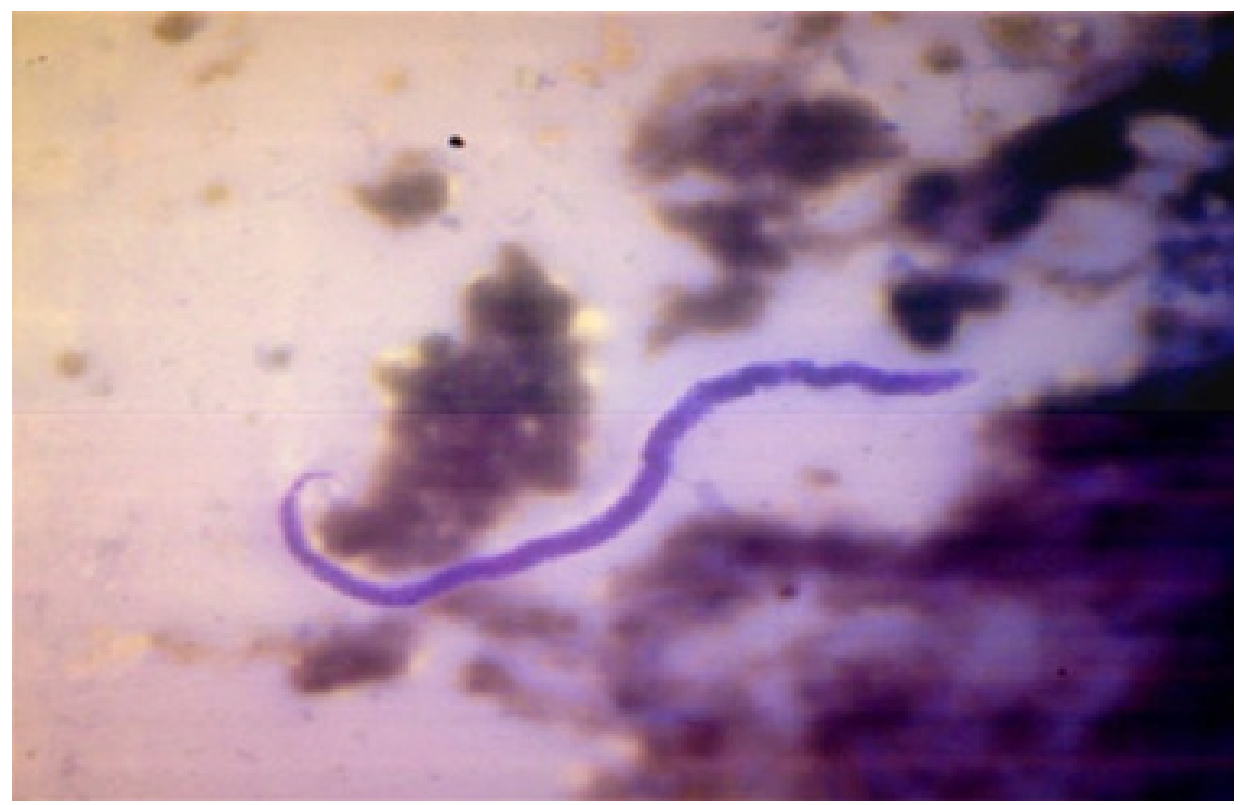

Figure1: Bone marrow aspirate showing microfilaria 


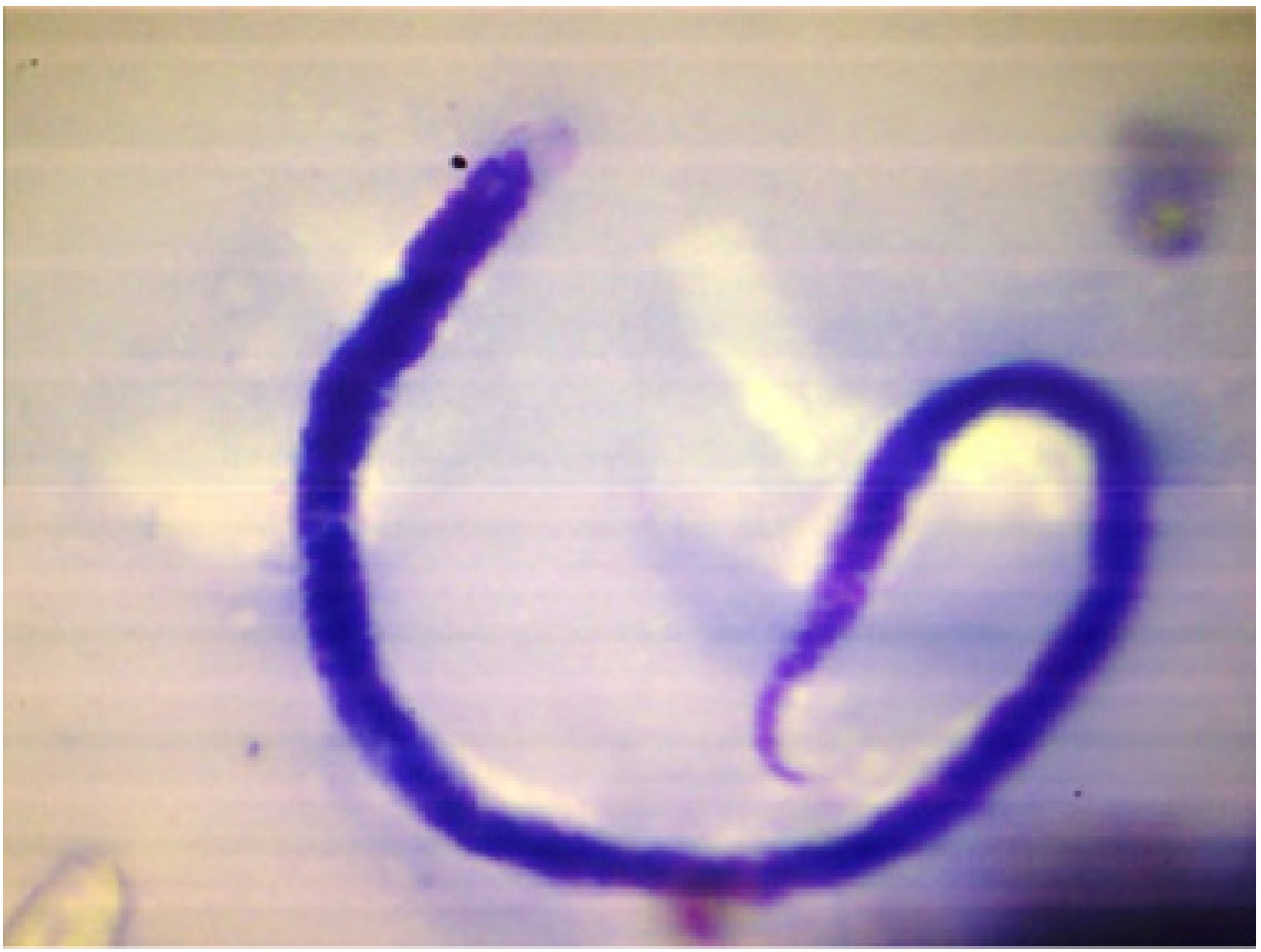

Figure 2: Microfilaria in bone marrow (high power view)

Patient was started on DEC $100 \mathrm{mg}$ for three weeks and albendazole 400mg twice daily for two weeks. His fever subsided in two days and his blood counts started improving (total count 1800/ $\mu \mathrm{l} \&$ hemoglobin $7.4 \mathrm{~g} \%$ ). He was discharged and kept under follow up. On review after one month, he is totally asymptomatic with normal blood counts and hemoglobin.

\section{Discussion}

Parasitic infestations are very common in the tropics causing several illnesses with varying presentations, and they escape our attention when they have atypical manifestations. Filariasis is disabling parasitic disease prevalent worldwide caused by various species of filarial organism. Bancroftian filariasis is infection by the filarial worm Wuchereria bancrofti which causes disease by blocking lymphatic vessels [1]. W. bancrofti is responsible for $90.0 \%$ of cases of filariasis and is found throughout the tropics and in some sub-tropical areas world-wide. Brugia malayi is confined to South-east and Eastern Asia. Bancroftian filariasis produce wide spectrum of clinical manifestations. The acute phase is characterized by fever, lymphangitis, lymphadenitis, epididymo-orchitis, and funiculitis. Headache, backache, muscle pain, insomnia, anorexia, urticarial rash, malaise, nausea and fatigue are common complaints. Eosinophilia and microfilaremia are common in acute phase. Chronic stage of bancroftian filariasis is characterized by lymphadenopathy, lymphadema, hydrocele, and elephantiasis [1].

A review of literature reveals detection of microfilaria in most of the commonly performed cytological specimens and mostly they are incidental. Microfilaria have been detected in cervicovaginal smears, endometrial smears, nipple secretions, ovarian cyst fluid, breast aspirates, hydrocele fluid, epididymal aspirates, urine samples, lung aspirates, pleural fluid, bronchial washings, ascitic fluid, intraoperative peritoneal fluid, lymph node aspirates, thyroid aspirates, salivary gland aspirates, bronchial brushings, laryngeal and pharyngeal brushings, gastric brushings, pericardial fluid, cutaneous nodule, soft tissue nodule, oral ulcer, bone marrow aspirates [2], brain aspirates and joint aspirates. Association of microfilariae with debilitating conditions suggests that it is an opportunistic infection [3]. Association of filariasis with neoplasm is often seen, although the role in tumorogenesis is controversial. It is very uncommon to get bone marrow failure associated with microfilaria. In our patient, there was a prompt response to DEC and Albendazole with complete recovery. So, in endemic areas, filariasis along with other parasitic infestations also should also be considered as differential diagnosis in bone marrow failure associated with fever. The hypoplasia could have been focal or it could have been a secondary Myelodysplastic syndrome resulting from parasitic infestations and probable cytokine mediated bone marrow failure.

\section{References}

1. Meyers WM, Neafi RC, Connor DH (1976) Diseases caused by filarial Nematodes-Bancroftian and Malayan Filariasis Pathology of Tropical and Extraordinary diseases. An Atlas. Vol II. Washington DC: Armed Forces Institute of Pathology: 340-55.

2. Sharma S, Rawat A, Chowhan A (2006) Microfilaria in bone marrow aspiration smears, correlation with marrow hypoplasia: a report of six cases. Indian J Pathol Microbiol 49: 566-8.

3. Gupta K, Sehgal A, Puri MM, Sidhwa HK (2002) Microfilariae in association with other diseases. A report of six cases. Acta Cytol 46: 776-8. 


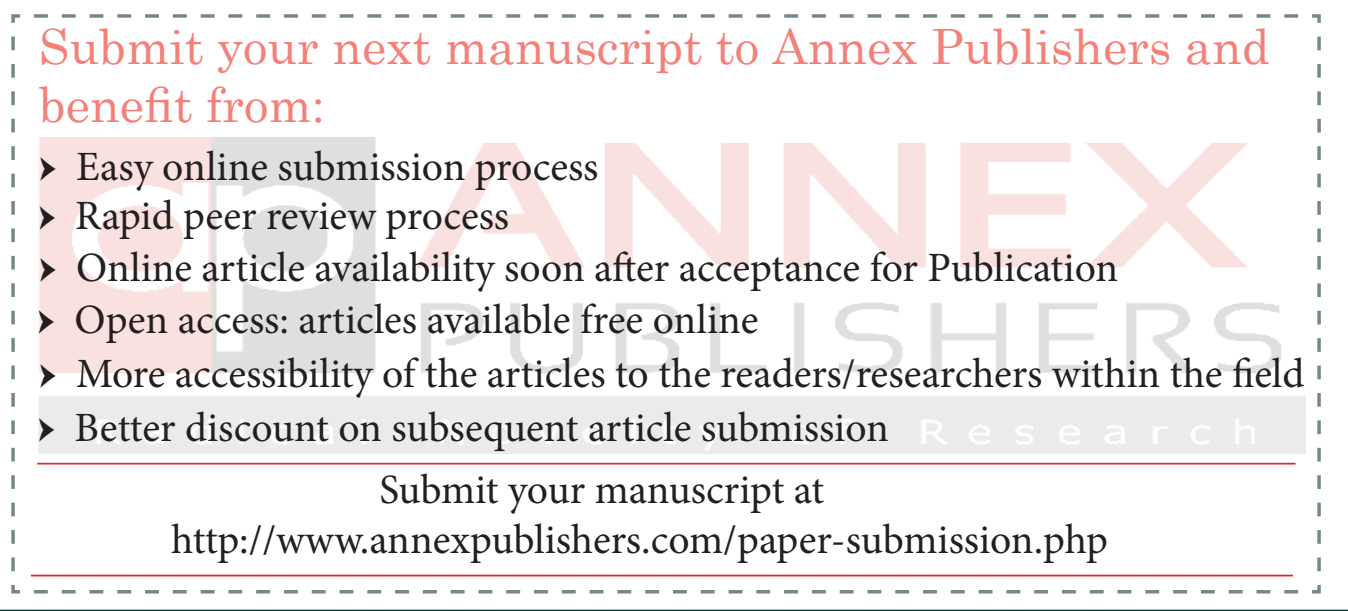

